Internat. J. Math. \& Math. Sci.

Vol. 22, No. 4 (1999) 765-773

S 0161-1712<99>22765-2

(c) Electronic Publishing House

\title{
SUFFICIENCY FOR GAUSSIAN HYPERGEOMETRIC FUNCTIONS TO BE UNIFORMLY CONVEX
}

\author{
YONG CHAN KIM and S. PONNUSAMY
}

(Received 10 July 1997 and in revised form 27 October 1997)

\begin{abstract}
Let $F(a, b ; c ; z)$ be the classical hypergeometric function and $f$ be a normalized analytic functions defined on the unit disk $U$. Let an operator $I_{a, b ; c}(f)$ be defined by $\left[I_{a, b ; c}(f)\right](z)=z F(a, b ; c ; z) * f(z)$. In this paper the authors identify two subfamilies of analytic functions $\mathscr{F}_{1}$ and $\mathscr{F}_{2}$ and obtain conditions on the parameters $a, b, c$ such that $f \in \mathscr{F}_{1}$ implies $I_{a, b ; c}(f) \in \mathscr{F}_{2}$.
\end{abstract}

Keywords and phrases. Uniformly convex function, Gaussian hypergeometric function.

1991 Mathematics Subject Classification. 30C45, 33C20.

1. Introduction. Let $\mathscr{A}$ denote the class of all normalized analytic functions $f(z)$ in the unit disc $\mathcal{U}=\{z \in \mathbb{C}:|z|<1\}$ of the form

$$
f(z)=z+\sum_{n=2}^{\infty} a_{n} z^{n} .
$$

Denote by $\mathscr{S}$ the class of all functions in $\mathscr{A}$ which are univalent in $\mathcal{U}$. For $\beta<1$ and real $\eta$, we let

$$
R_{\eta}(\beta):=\left\{f \in \mathscr{A}: \operatorname{Re}\left[e^{i \eta}\left(f^{\prime}(z)-\beta\right)\right]>0, z \in U\right\} .
$$

It is a well-known result that when $\beta \geq 0$ we have $R_{\eta}(\beta) \subset \mathscr{S}$ and for $\beta<0$, the functions in $R_{\eta}(\beta)$ need not be univalent in $U$. A function $f$ is said to be uniformly convex (UCV) if the image of every circular arc $\gamma$ contained in $U$, with center also in $U$, is convex. Analytically, UCV family is characterized as follows:

$$
\mathrm{UCV}=\left\{f \in \mathscr{S}:\left|\frac{z f^{\prime \prime}(z)}{f^{\prime}(z)}\right| \leq \operatorname{Re}\left\{1+\frac{z f^{\prime \prime}(z)}{f^{\prime}(z)}\right\}, z \in \mathcal{U}\right\},
$$

see $[8]$.

For $\alpha \geq 0$, let $\operatorname{UCT}(\alpha)$ denote the subfamily of functions $f(z)=z-\sum_{n=2}^{\infty}\left|a_{n}\right| z^{n}$ in $\mathscr{A}$ which satisfy the condition [11]

$$
\operatorname{Re}\left\{1+\frac{z f^{\prime \prime}(z)}{f^{\prime}(z)}\right\} \geq \alpha\left|\frac{z f^{\prime \prime}(z)}{f^{\prime}(z)}\right|, \quad z \in u,
$$

for some $\alpha \geq 0$. In this paper we are mainly interested in the Gaussian hypergeometric function $F(a, b ; c ; z)$ defined by

$$
F(a, b ; c ; z):=\sum_{n=0}^{\infty} \frac{(a)_{n}(b)_{n}}{(c)_{n}} \cdot \frac{z^{n}}{n !},
$$

where $a, b, c \in \mathbb{C}$ with $c \neq 0,-1,-2, \ldots$. Here $(a)_{0}:=1$ for $a \neq 0$ and if $n$ is a positive 
integer, $(a)_{n}$ denotes the ascending factorial notation $(a)_{n}:=a(a+1) \cdots(a+n-1)$. In the exceptional case $c=-p$, where $p$ is a positive integer, $F(a, b ; c ; z)$ is defined if $a=-m$ or $b=-m$, where $m=0,1,2, \ldots$ and $m \leq p$. We also note that if $a=-m$, then we have $(-m)_{n}=0$ for all $n \geq m+1$ and therefore in this case $F(a, b ; c ; z)$ becomes a polynomial of degree $m$. This observation is to indicate that our main results (see Theorems 2 and 3(iv)) give also geometrical information for polynomials. It is important to point out that the hypergeometric function $F(a, b ; c ; z)$ can be classified into three cases according to whether $\operatorname{Re}(c-a-b)$ equals zero, negative, or positive. In the last case, the function $F(a, b ; c ; z)$ is bounded in $U$ whereas the case $\operatorname{Re}(c-a-b) \leq 0$, it is unbounded in $U$ as it has a pole at $z=1$ in this case. The asymptotic behaviour of the hypergeometric function $F(a, b ; c ; x)$ for $\operatorname{Re}(c-a-b) \leq 0$ near the singularity $x \rightarrow 1$ has been studied in detail by Ponnusamy and Vuorinen [7] and thus improving several known results in the literature, see [2] or [12, p. 299].

For $f \in \mathscr{A}$, we recall the operator $I_{a, b ; c}(f)$ of Hohlov [3], which maps $\mathscr{A}$ into itself, defined by

$$
\left[I_{a, b ; c}(f)\right](z)=z F(a, b ; c ; z) * f(z),
$$

where $*$ denotes the usual Hadamard product (convolution) of power series. When $f(z)$ equals the convex function $z /(1-z)$, then the operator $I_{a, b ; c}(f)$ in this case becomes $z F(a, b ; c ; z)$. We also note that the operator $I_{a, b ; c}(f)$ is a natural choice for studying the geometric properties of it because of its interaction with geometric function theory for the special operator popularly known as Bernardi operator. In fact the Bernardi operator is a special case of the convolution operator $I_{a, b ; c}(f)$ when $a=1, b=1+\gamma, c=2+\gamma$ with $\operatorname{Re} \gamma>-1$ :

$$
\left[I_{1,1+\gamma ; 2+\gamma}(f)\right](z):=B_{f}(z)=\frac{1+\gamma}{z^{\gamma}} \int_{0}^{z} t^{\gamma-1} f(t) d t .
$$

Here $I_{1,1 ; 2}(f)$ and $I_{1,2 ; 3}(f)$ are known as Alexander and Liberia operators, respectively. In [3] Hohlov determined the conditions to guarantee that $I_{a, b ; c}(f)$ is univalent in $u$ for a function $f$ in $\mathscr{Y}$.

In this paper, we consider the following problem: for a given $a, b, c$ such that $\operatorname{Re}(c-$ $a-b)>0$, we find conditions such that $z F(a, b ; c ; z) \in \operatorname{UCV}$ or $\operatorname{UCT}(\alpha)$. We also find conditions such that $I_{a, b ; c}(f) \in \mathrm{UCV}$ if $f \in R_{\eta}(\beta)$.

2. Preliminary results. By using the Gauss summation theorem [9, p. 19, eq. (20)], we immediately have Lemmas 1 and 2

LEMmA 1 [6, Lem. 3.2]. Let $a \in \mathbb{C} \backslash\{0\}, c>\max \{0,2+2 \operatorname{Re} a\}$, and

$$
S=\sum_{n=0}^{\infty} \frac{(n+1)^{2}\left|(a)_{n}\right|^{2}}{(c)_{n}(1)_{n}}
$$

Then we have

$$
S=\frac{\Gamma(c-2 \operatorname{Re} a) \Gamma(c)}{\Gamma(c-a) \Gamma(c-\bar{a})}\left[1+\frac{\left|(a)_{2}\right|^{2}}{(c-2-2 \operatorname{Re} a)_{2}}+\frac{3|a|^{2}}{c-1-2 \operatorname{Re} a}\right] .
$$


LEMmA 2 [6, Lem. 3.3]. Let $a, b \in \mathbb{C} \backslash\{0\}, c>0$. Then we have the following:

(i) For $a, b>0, c>a+b+1$,

$$
\sum_{n=0}^{\infty} \frac{(n+1)(a)_{n}(b)_{n}}{(c)_{n}(1)_{n}}=\frac{\Gamma(c-a-b) \Gamma(c)}{\Gamma(c-a) \Gamma(c-b)}\left[\frac{a b}{c-1-a-b}+1\right] .
$$

(ii) For $a, b>0, c>a+b+2$,

$$
\sum_{n=0}^{\infty} \frac{(n+1)^{2}(a)_{n}(b)_{n}}{(c)_{n}(1)_{n}}=\frac{\Gamma(c-a-b) \Gamma(c)}{\Gamma(c-a) \Gamma(c-b)}\left[1+\frac{(a)_{2}(b)_{2}}{(c-2-a-b)_{2}}+\frac{3 a b}{c-1-a-b}\right] .
$$

(iii) For $a \neq 1, b \neq 1$, and $c \neq 1$ with $c>\max \{0, a+b-1\}$,

$$
\sum_{n=0}^{\infty} \frac{(a)_{n}(b)_{n}}{(c)_{n}(1)_{n+1}}=\frac{1}{(a-1)(b-1)}\left[\frac{\Gamma(c+1-a-b) \Gamma(c)}{\Gamma(c-a) \Gamma(c-b)}-(c-1)\right] .
$$

(iv) For $a \neq 1$ and $c \neq 1$ with $c>\max \{0,2 \operatorname{Re} a-1\}$,

$$
\sum_{n=0}^{\infty} \frac{\left|(a)_{n}\right|^{2}}{(c)_{n}(1)_{n+1}}=\frac{1}{|a-1|^{2}}\left[\frac{\Gamma(c+1-2 \operatorname{Re} a) \Gamma(c)}{\Gamma(c-a) \Gamma(c-\bar{a})}-(c-1)\right] .
$$

(v) For $a, b>0, c>a+b+3$,

$$
\begin{aligned}
& \sum_{n=0}^{\infty} \frac{(n+1)^{3}(a)_{n}(b)_{n}}{(c)_{n}(1)_{n}} \\
& \quad=\frac{\Gamma(c-a-b) \Gamma(c)}{\Gamma(c-a) \Gamma(c-b)}\left[\frac{(a)_{3}(b)_{3}}{(c-3-a-b)_{3}}+\frac{6(a)_{2}(b)_{2}}{(c-2-a-b)_{2}}+\frac{7 a b}{c-1-a-b}+1\right] .
\end{aligned}
$$

(vi) For $c>\max \{0,2 \operatorname{Re} a+3\}$,

$$
\begin{aligned}
& \sum_{n=0}^{\infty} \frac{(n+1)^{3}\left|(a)_{n}\right|^{2}}{(c)_{n}(1)_{n+1}} \\
& \quad=\frac{\Gamma(c-2 \operatorname{Re} a) \Gamma(c)}{\Gamma(c-a) \Gamma(c-\bar{a})}\left[\frac{\left|(a)_{3}\right|^{2}}{(c-3-2 \operatorname{Re} a)_{3}}+\frac{6\left|(a)_{2}\right|^{2}}{(c-2-2 \operatorname{Re} a)_{2}}+\frac{7|a|^{2}}{c-1-2 \operatorname{Re} a}+1\right] .
\end{aligned}
$$

Our main results rely on the following lemmas:

LEMMA 3 [11, Thm. 1]. If $\sum_{n=2}^{\infty} n(2 n-1)\left|a_{n}\right| \leq 1$, then the function of the form (1.1) is in UCV.

LEMMA 4. Let the function $f(z)$ be of the form (1.1). Then a sufficient condition for $f$ to satisfy $\operatorname{Re} e^{i n}(f(z) / z-\beta)>0$ in $u$ is

$$
\sum_{n=2}^{\infty}\left|a_{n}\right| \leq(1-\beta) \cos \eta \quad\left(|\eta|<\frac{\pi}{2}, \beta<1\right) .
$$

The condition (2.9) is also necessary if $\eta=0$, and $a_{n}<0$ for all $n \geq 2$. 
Proof. The proof is immediate because

$$
\begin{aligned}
\operatorname{Re} e^{i \eta}\left(\frac{f(z)}{z}-\beta\right) & =(1-\beta) \cos \eta+\operatorname{Re}\left(e^{i \eta} \sum_{n=2}^{\infty} a_{n} z^{n-1}\right) \\
& \geq(1-\beta) \cos \eta-\sum_{n=2}^{\infty}\left|a_{n}\right||z|^{n-1} \\
& \geq(1-\beta) \cos \eta-\sum_{n=2}^{\infty}\left|a_{n}\right| \geq 0
\end{aligned}
$$

The necessary part (with $\eta=0$ ) follows upon taking $z \rightarrow 1$, since $a_{n}<0$ in this case.

3. Main results. We begin by proving the following theorem:

THEOREM 1. Let $a, b \in \mathbb{C} \backslash\{0\}$, and $c>|a|+|b|+2$. Then a sufficient condition for the function $z F(a, b ; c ; z)$ belong to $U C V$ is that

$$
\frac{\Gamma(c-|a|-|b|) \Gamma(c)}{\Gamma(c-|a|) \Gamma(c-|b|)}\left[1+\frac{2(|a|)_{2}(|b|)_{2}}{(c-2-|a|-|b|)_{2}}+\frac{5|a b|}{c-|a|-|b|-1}\right] \leq 2 .
$$

Proof. Set $f(z)=z F(a, b ; c ; z)$. Then, by using Lemma 3 , it suffices to show that

$$
T:=\sum_{n=1}^{\infty}(n+1)(2 n+1)\left|\frac{(a)_{n}(b)_{n}}{(c)_{n}(1)_{n}}\right| \leq 1 .
$$

From the fact that $\left|(a)_{n}\right| \leq(|a|)_{n}$, we observe that

$$
\begin{aligned}
T & \leq \sum_{n=1}^{\infty}(n+1)(2 n+1) \frac{(|a|)_{n}(|b|)_{n}}{(c)_{n}(1)_{n}} \\
& =2 \sum_{n=0}^{\infty}(n+1)^{2} \frac{(|a|)_{n}(|b|)_{n}}{(c)_{n}(1)_{n}}-\sum_{n=0}^{\infty}(n+1) \frac{(|a|)_{n}(|b|)_{n}}{(c)_{n}(1)_{n}}-1 \\
& =\frac{\Gamma(c-|a|-|b|) \Gamma(c)}{\Gamma(c-|a|) \Gamma(c-|b|)}\left[1+\frac{2(|a|)_{2}(|b|)_{2}}{(c-2-|a|-|b|)_{2}}+\frac{5|a b|}{c-|a|-|b|-1}\right]-1,
\end{aligned}
$$

by (i) and (ii) of Lemma 2. Hence, by the condition (3.1), $T$ is less than 1 . This completes the proof.

If, in the proof of Theorem 1 , we start with $b=\bar{a}$, then we have the following theorem under a weaker condition on $c$ :

TheOREM 2. Let $a \in \mathbb{C} \backslash\{0\}, c>\max \{2+2 \operatorname{Re} a, 0\}$, and

$$
\frac{\Gamma(c-2 \operatorname{Re} a) \Gamma(c)}{\Gamma(c-a) \Gamma(c-\bar{a})}\left[1+\frac{2\left|(a)_{2}\right|^{2}}{(c-2-2 \operatorname{Re} a)_{2}}+\frac{5|a|^{2}}{c-1-2 \operatorname{Re} a}\right] \leq 2 .
$$

Then $z F(a, \bar{a} ; c ; z) \in U C V$.

PROoF. The proof of this theorem follows in the similar lines of proof of Theorem 1 if we use Lemma 1 and therefore we omit the details. 
EXAMPLE 1. If we take $a=-2$ in Theorem 2, then the conditions on $c$ in Theorem 2 become $c \geq((23+\sqrt{745}) / 2)$. This observation gives the following conclusion: for $c \geq((23+\sqrt{745}) / 2)$, the function

$$
z F(-2,-2 ; c ; z)=z+\frac{4}{c} z^{2}+\frac{2}{c(c+1)} z^{3}
$$

is in the class UCV.

In this way one can easily construct a higher order polynomial function lying in the UCV class.

By using Lemma 4, we obtain

THEOREM 3. Suppose that $a, b, c$ and $\beta<1$ are related by any one of the following conditions:

(i) $a, b>0, c>a+b$, and

$$
\frac{\Gamma(c-a-b) \Gamma(c)}{\Gamma(c-a) \Gamma(c-b)}-1 \leq(1-\beta) \cos \eta
$$

(ii) $-1<a<0, b>0, c>b$, and

$$
\beta \leq 1-\frac{1}{\cos \eta}\left[1-\frac{\Gamma(c-a-b) \Gamma(c)}{\Gamma(c-a) \Gamma(c-b)}\right]
$$

(iii) $a, b \in \mathbb{C} \backslash\{0\}, c>|a|+|b|$, and

$$
\frac{\Gamma(c-|a|-|b|) \Gamma(c)}{\Gamma(c-|a|) \Gamma(c-|b|)}-1 \leq(1-\beta) \cos \eta ;
$$

(iv) $a \in \mathbb{C} \backslash\{0\}, b=\bar{a}, c>\max \{0,2 \operatorname{Re} a\}$, and

$$
\frac{\Gamma(c-2 \operatorname{Re} a) \Gamma(c)}{\Gamma(c-a) \Gamma(c-\bar{a})}-1 \leq(1-\beta) \cos \eta
$$

Then we have Re $e^{i \eta}(F(a, b ; c ; z)-\beta)>0$ for $z \in \mathcal{U}$.

Proof. By Lemma 4, it suffices to show that

$$
\sum_{n=1}^{\infty}\left|\frac{(a)_{n}(b)_{n}}{(c)_{n}(1)_{n}}\right| \leq(1-\beta) \cos \eta
$$

First, we recall the well-known formula (cf. [9, p. 19, eq. (20)])

$$
F(a, b ; c ; 1)=\frac{\Gamma(c-a-b) \Gamma(c)}{\Gamma(c-a) \Gamma(c-b)} \quad(\operatorname{Re}(c-a-b)>0),
$$

If $a, b>0$ and $c>a+b$, then from using this formula we have

$$
\sum_{n=1}^{\infty} \frac{(a)_{n}(b)_{n}}{(c)_{n}(1)_{n}}=\frac{\Gamma(c-a-b) \Gamma(c)}{\Gamma(c-a) \Gamma(c-b)}-1 .
$$

Similarly, if $-1<a<0, b>0$ and $c>b$ then, by Lemma 2 and the formula (3.11), we obtain that

$$
\sum_{n=1}^{\infty}\left|\frac{(a)_{n}(b)_{n}}{(c)_{n}(1)_{n}}\right|=\frac{|a b|}{c} \sum_{n=0}^{\infty} \frac{(a+1)_{n}(b+1)_{n}}{(c+1)_{n}(1)_{n+1}}=1-\frac{\Gamma(c-a-b) \Gamma(c)}{\Gamma(c-a) \Gamma(c-b)} .
$$

Thus under the conditions (3.6) and (3.7), the conclusion follows. 
On applying the ideas of the proofs of Theorems 1 and 2, we can obtain the required conclusion by assuming the conditions (iii) and (iv), respectively. Therefore we complete the proof.

REMARK 1. From Lemma 4, we observe that the condition (3.7) for $\eta=0$ is necessary and sufficient for $\operatorname{Re} F(a, b ; c ; z)>\beta$.

Now, we consider the incomplete Beta function $\phi(a, c ; z)$ which is defined by

$$
\phi(a, c ; z):=z F(1, a ; c ; z)=\sum_{n=0}^{\infty} \frac{(a)_{n}}{(c)_{n}} z^{n+1} \quad(c \neq 0,-1,-2, \ldots, z \in U) .
$$

Corresponding to the function $\phi(a, c ; z)$, Carlson and Shaffer [1] defined a linear operator $\mathscr{L}(a, c)$ on $\mathscr{A}$ by the convolution [1, p. 738 eq. (2.2)]:

$$
\mathscr{L}(a, c) f(z)=\phi(a, c ; z) * f(z) \quad(f \in \mathscr{A}) .
$$

Clearly,

$$
\mathscr{L}(a, c)=I_{a, 1 ; c} .
$$

We note that $\phi^{\prime}(a, c ; z)=F(a, 2 ; c ; z)$. Therefore if we take $b=2$ in Theorem 3 , then by using this observation we have the following corollary:

COROLLARY 1. Suppose that $a, c$, and $\beta<1$ are related by any one of the following conditions:

(i) $a>0, c>a+2$, and

$$
\frac{(c-1)(c-2)}{(c-a-1)(c-a-2)} \leq 1+(1-\beta) \cos \eta
$$

(ii) $-1<a<0, c>2$, and

$$
\beta \leq 1-\frac{1}{\cos \eta}\left[1-\frac{(c-1)(c-2)}{(c-a-1)(c-a-2)}\right]
$$

(iii) $a \in \mathbb{C} \backslash\{0\}, c>|a|+2$, and

$$
\frac{(c-1)(c-2)}{(c-|a|-1)(c-|a|-2)} \leq 1+(1-\beta) \cos \eta .
$$

Then we have $\operatorname{Re} e^{i \eta}\left(\phi^{\prime}(a, c ; z)-\beta\right)>0$ in $u$.

EXAMPLE 2. From (ii) of Corollary 1 we obtain the following sharp result:

$$
\operatorname{Re} \phi^{\prime}(a, c ; z)>\frac{(c-1)(c-2)}{(c-a-1)(c-a-2)}
$$

for $a \in(-1,0)$ and $c>2$. This is an improvement of a recent result in [4, Thm. 4].

Next, we establish the following corollary which deals with convolution of functions having their real parts in the half-plane.

COROLlary 2. Let anyone of conditions (i)-(iv) of Theorem 3 be satisfied with $\eta=0$. Then we have

$$
f \in R_{\eta}\left(\beta_{1}\right) \Longrightarrow I_{a, b ; c}(f) \in R_{\eta}\left(\beta_{2}\right),
$$


where $\beta_{2}$ is given by

$$
1-\beta_{2}=2\left(1-\beta_{1}\right)(1-\beta)
$$

and $\beta$ is as in Theorem 3.

Proof. Define $g(z)=\left[I_{a, b ; c}(f)\right](z)=z F(a, b ; c ; z) * f(z)$. Then it is clear that

$$
g^{\prime}(z)=F(a, b ; c ; z) * f^{\prime}(z) .
$$

By hypothesis Re $e^{i \eta}\left(f^{\prime}(z)-\beta_{1}\right)>0$ and $\operatorname{Re} F(a, b ; c ; z)>\beta$. Therefore, using Lemma in [5] (see also [10] for the case $\eta=0$ ), we see that $\operatorname{Re} e^{i \eta}\left(g^{\prime}(z)-\beta_{2}\right)>0$, where $\beta_{2}$ is defined by (3.22). This completes the proof.

By Lemma 3, we obtain

THEOREM 4. If $a, b \in \mathbb{C} \backslash\{0\}, c>|a|+|b|+1$ satisfy the condition

$$
2(1-\beta) \cos \eta\left[\frac{\Gamma(c-|a|-|b|) \Gamma(c)}{\Gamma(c-|a|) \Gamma(c-|b|)}\left(\frac{2|a b|}{c-|a|-|b|-1}+1\right)-1\right] \leq 1,
$$

then the operator $I_{a, b ; c}(f)$ maps $R_{\eta}(\beta)$ into $U C V$.

Proof. Suppose that the function $f(z)$, defined by (1.1), is in the class $R_{\eta}(\beta)$. Then by using the standard technique, we can directly get the coefficient estimate

$$
\left|a_{n}\right| \leq \frac{2}{n}(1-\beta) \cos \eta
$$

(see [6, eq. (4.1)]). Hence, by Lemma 3, it is enough to show that

$$
2(1-\beta) \cos \eta \sum_{n=1}^{\infty}(2 n-1)\left|\frac{(a)_{n}\left(b_{n}\right)}{(c)_{n}(1)_{n}}\right| \leq 1 .
$$

If we use the hypotheses, this verification is very similar to that of Theorem 3 and therefore we omit the details.

Corollary 3. Suppose that $a \in \mathbb{C} \backslash\{0\}, c>\max \{0,1+2 \operatorname{Re} a\}$, and satisfies the condition

$$
2(1-\beta) \cos \eta\left[\frac{\Gamma(c-2 \operatorname{Re} a) \Gamma(c)}{\Gamma(c-a) \Gamma(c-\bar{a})}\left(\frac{2|a|^{2}}{c-2 \operatorname{Re} a-1}+1\right)-1\right] \leq 1 .
$$

Then the operator $I_{a, \bar{a} ; c}(f)$ maps $R_{\eta}(\beta)$ into $U C V$.

Making use of Lemmas 2 and 3, we obtain the following theorem:

THEOREM 5. Let $-1<a<0, b>0$, and $c>a+b+1$. Then a necessary and sufficient condition for $z F(a, b ; c ; z)$ to belong to $\operatorname{UCT}(\alpha)$ is that

$$
a+b+1+\left[\frac{(1+\alpha)(a+1)(b+1)}{c-a-b-2}+3+2 \alpha\right]|a b| \leq c .
$$

Proof. We write

$$
f(z)=z F(a, b ; c ; z)=z+\sum_{n=2}^{\infty} a_{n} z^{n}
$$


so that, by Lemma 3, it suffices to prove that

$$
S:=\sum_{n=1}^{\infty}(n+1)[(1+\alpha)(n+1)-\alpha]\left|a_{n+1}\right| \leq 1,
$$

where

$$
a_{n+1}=\frac{(a)_{n}(b)_{n}}{(c)_{n}(1)_{n}}
$$

Now

$$
\begin{aligned}
S=\sum_{n=1}^{\infty}(n+1)[(1+\alpha)(n+1)-\alpha]\left|\frac{(a)_{n}(b)_{n}}{(c)_{n}(1)_{n}}\right| \\
=\frac{|a b|}{c}\left[\left\{(1+\alpha) \sum_{n=0}^{\infty}(n+1) \frac{(a+1)_{n}(b+1)_{n}}{(c+1)_{n}(1)_{n}}\right\}\right. \\
\left.+(2+\alpha)\left\{\sum_{n=0}^{\infty} \frac{(a+1)_{n}(b+1)_{n}}{(c+1)_{n}(1)_{n}}\right\}+\sum_{n=0}^{\infty} \frac{(a+1)_{n}(b+1)_{n}}{(c+1)_{n}(1)_{n+1}}\right] .
\end{aligned}
$$

Using Lemma 2((i), (iii)), and the formula (3.11), we find that the sum $S$ can be simplified so that

$$
S=\frac{\Gamma(c-a-b-1) \Gamma(c)}{\Gamma(c-a) \Gamma(c-b)}\left[\left\{\frac{(1+\alpha)(a+1)(b+1)}{c-a-b-2}+3+2 \alpha\right\}|a b|-(c-a-b-1)\right]+1
$$

which, by the condition (3.28), gives $S \leq 1$. This completes the proof.

ACKNOWLEDGEMENT. This work was partially supported by KOSEF (94-0701-0201-3) and TGRC-KOSEF, and by the Korean Ministry of Education through Research Fund (BSRI-97-1401).

\section{REFERENCES}

[1] B. C. Carlson and D. B. Shaffer, Starlike and prestarlike hypergeometric functions, SIAM J. Math. Anal. 15 (1984), no. 4, 737-745. MR 85j:30014. Zbl 567.30009.

[2] R. J. Evans, Ramanujan's second notebook: asymptotic expansions for hypergeometric series and related functions, Ramanujan revisited (Boston, MA), Illinois University, Academic Press, 1988, pp. 537-560. MR 89c:33003. Zbl 646.33003.

[3] Y. E. Hohlov, Convolution operators preserving univalent functions, Pliska Stud. Math. Bulgar. 10 (1989), 87-92. MR 92h:30036. Zbl 829.30007.

[4] Y. C. Kim and H. M. Srivastava, Fractional integral and other linear operators associated with the Gaussian hypergeometric function, Complex Variables Theory Appl. 34 (1997), no. 3, 293-312. Zbl 980.39265.

[5] S. Ponnusamy, Inclusion theorems for convolution product of second order polylogarithms and functions with the derivative in a half plane, Rocky Mountain J. Math. 28 (1998), no. 2, 695-733. CMP 1651 594. Zbl 915.3002.

[6] S. Ponnusamy and F. Rønning, Geometric properties for convolutions of hypergeometric series with univalent and nonunivalent functions, Dept. of Math., Univ. of Helsinki 113 (1996), Preprint.

[7] S. Ponnusamy and M. Vuorinen, Asymptotic expansions and inequalities for hypergeometric function, Mathematika 44 (1997), no. 2, 278-301. Zbl 897.33001. 
[8] F. Rønning, Uniformly convex functions and a corresponding class of starlike functions, Proc. Amer. Math. Soc. 118 (1993), no. 1, 189-196. MR 93f:30017. Zbl 805.30012.

[9] H. M. Srivastava and P. W. Karlsson, Multiple Gaussian hypergeometric series, Ellis Horwood Series: Mathematics and its Applications, Halsted Press: a division of John Wiley \& Sons, New York, Chichester, Brisbane, Toronto, 1985. MR 87f:33015. Zbl 552.33001 .

[10] J. Stankiewicz and Z. Stankiewicz, Some applications of the Hadamard convolution in the theory of functions, Ann. Univ. Mariae Curie-Skłodowska Sect. A 40 (1986), 251-265, Proceedings of the 9th Conference on Analytic Functions. MR 89e:30030. Zbl 642.30019 .

[11] K. G. Subramanian, G. Murugusundaramoorthy, P. Balasubrahmanyam, and H. Silverman, Subclasses of uniformly convex and uniformly starlike functions, Math. Japon. 42 (1995), no. 3, 517-522. MR 96h:30018. Zbl 837.30011.

[12] E. T. Whittaker and G. N. Watson, A course of modern analysis. An introduction to the general theory of infinite processes and of analytic functions: with an account of the principal transcendental functions, 4th ed., Cambridge University Press, New York, 1962, An introduction to the general theory on infinite processes and of analytic functions; with an account of the principal transcendental functions. MR 31\#2375. Zbl 105.26901.

Kim: Department of Mathematics, Yeungnam University, 214-1, DAedOnG, GyongSAN 712-749, KOREA

Ponnusamy: Department of Mathematics, University of Helsinki, P. O. BoX 4, HallitSKATU 15, FIN-00014, HELSINKI, FINLAND 


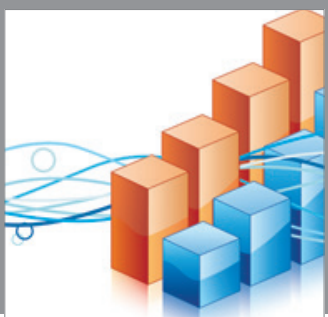

Advances in

Operations Research

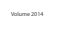

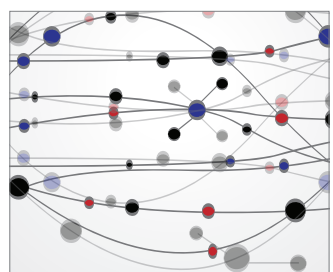

\section{The Scientific} World Journal
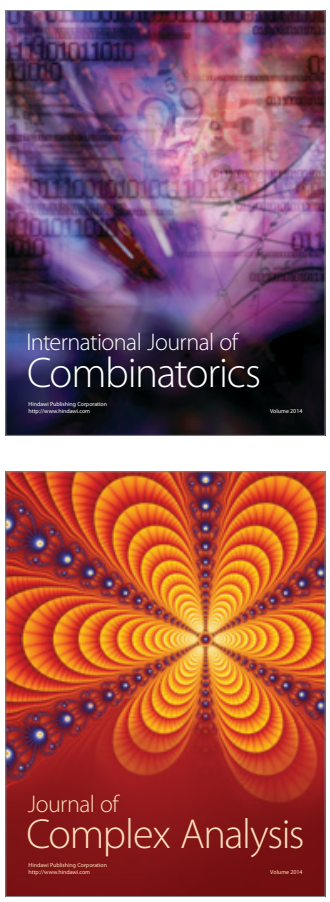

International Journal of

Mathematics and

Mathematical

Sciences
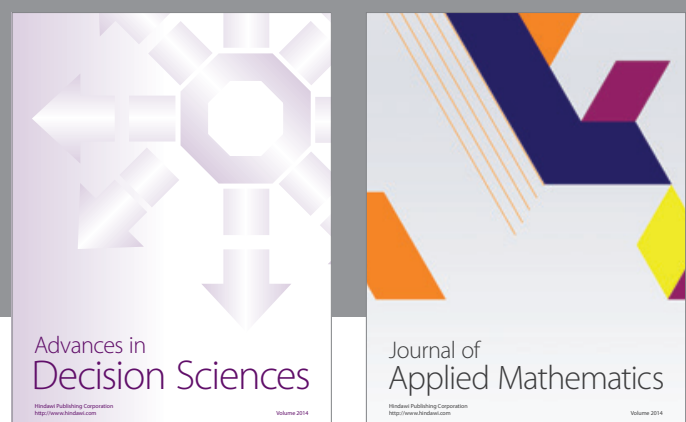

Journal of

Applied Mathematics
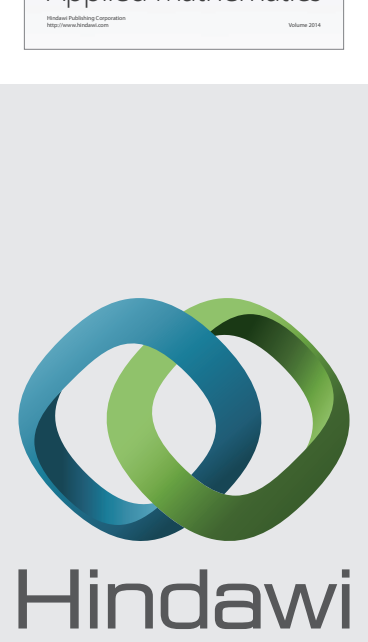

Submit your manuscripts at http://www.hindawi.com
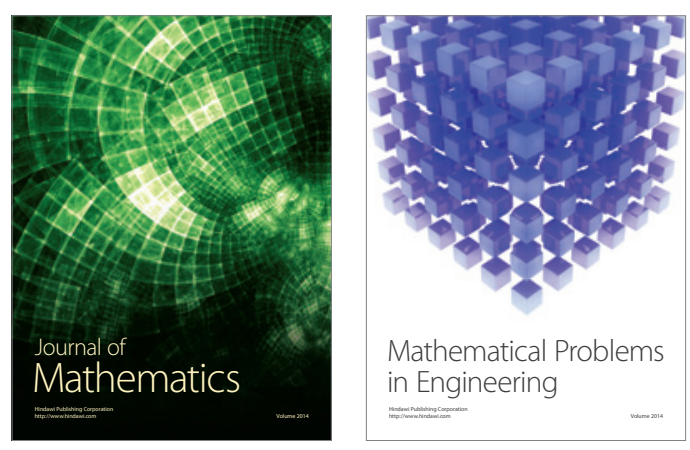

Mathematical Problems in Engineering
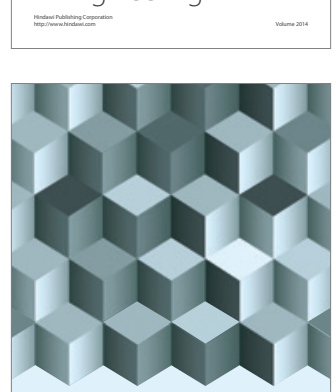

Journal of

Function Spaces
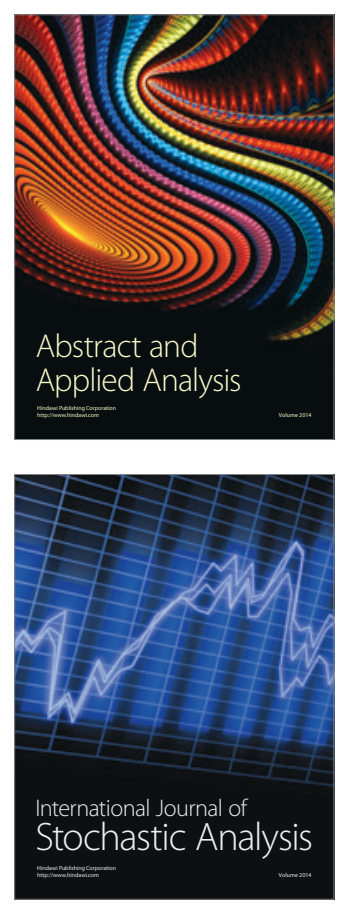

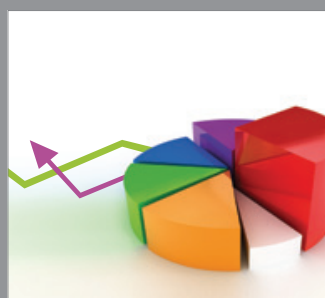

ournal of

Probability and Statistics

Promensencen
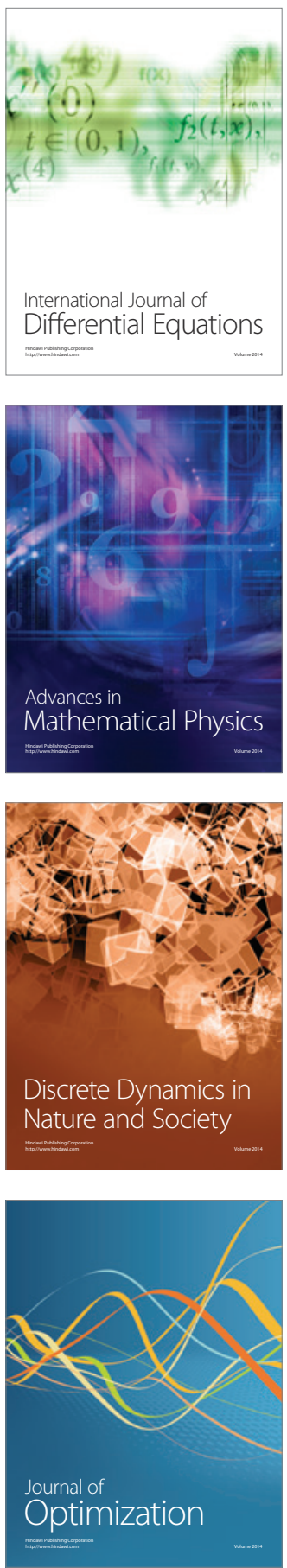